\title{
Endothelial lipase is a major determinant of HDL level
}

\author{
Tatsuro Ishida, ${ }^{1,3}$ Sungshin Choi, ${ }^{4}$ Ramendra K. Kundu, ${ }^{1}$ Ken-ichi Hirata, ${ }^{3}$ \\ Edward M. Rubin, ${ }^{5}$ Allen D. Cooper, ${ }^{2,4}$ and Thomas Quertermous ${ }^{1}$ \\ ${ }^{1}$ Division of Cardiovascular Medicine and Donald W. Reynolds Cardiovascular Clinical Research Center, and \\ ${ }^{2}$ Division of Gastroenterology, Stanford University School of Medicine, Stanford, California, USA \\ ${ }^{3}$ Division of Cardiovascular and Respiratory Medicine, Kobe University Graduate School of Medicine, Kobe, Japan \\ ${ }^{4}$ Research Institute, Palo Alto Medical Foundation, Palo Alto, California, USA \\ ${ }^{5}$ Genome Sciences Department, Lawrence Berkeley National Laboratory, Berkeley, California, USA
}

\begin{abstract}
A new member of the lipase gene family, initially termed endothelial lipase (gene nomenclature, $L I P G$; protein, EL), is expressed in a variety of different tissues, suggesting a general role in lipid metabolism. To assess the hypothesis that EL plays a physiological role in lipoprotein metabolism in vivo, we have used gene targeting of the native murine locus and transgenic introduction of the human LIPG locus in mice to modulate the level of EL expression. Evaluation of these alleles in a $\mathrm{C} 57 \mathrm{Bl} / 6$ background revealed an inverse relationship between HDL cholesterol level and EL expression. Fasting plasma HDL cholesterol was increased by $57 \%$ in $\mathrm{LIPG}^{-/-}$mice and $25 \%$ in $\mathrm{LIPG}^{+/-}$mice and was decreased by $19 \%$ in $L I P G$ transgenic mice as compared with syngeneic controls. Detailed analysis of lipoprotein particle composition indicated that this increase was due primarily to an increased number of HDL particles. Phospholipase assays indicated that EL is a primary contributor to phospholipase activity in mouse. These data indicate that expression levels of this novel lipase have a significant effect on lipoprotein metabolism.
\end{abstract}

J. Clin. Invest. 111:347-355 (2003). doi:10.1172/JCI200316306.

\section{Introduction}

Study of the association of serum lipid levels with atherosclerosis has led to the characterization of HDL level as an inverse correlate of the risk of coronary heart disease $(1,2)$. Up to $70 \%$ of the variation in HDL cholesterol levels in humans is genetically determined, but the genes contributing to this variation are incompletely defined $(3,4)$. It is generally postulated that variations in plasma HDL cholesterol levels are determined by both the rate at which HDL cholesterol is produced and the rate of catabolism of HDL particles $(5,6)$. There are several known molecules that play a role in regulating plasma HDL cholesterol levels, including hepatic lipase (HL), lecithin cholesterol acyltransferase (LCAT), cholesteryl ester transfer protein (CETP), and

Received for publication June 28, 2002, and accepted in revised form October 22, 2002.

Address correspondence to: Thomas Quertermous, Division of Cardiovascular Medicine, Stanford University School of Medicine, 300 Pasteur Drive, Falk CVRC, Stanford, California 94305, USA. Phone: (650) 723-5013; Fax: (650) 725-2178; E-mail: tomq1@stanford.edu.

Conflict of interest: The authors have declared that no conflict of interest exists.

Nonstandard abbreviations used: endothelial lipase gene (LIPG); endothelial lipase protein (EL); hepatic lipase (HL); lecithin cholesterol acyltransferase (LCAT), cholesteryl ester transfer protein (CETP); phospholipid transfer protein (PLTP); lipoprotein lipase (LPL); neomycin phosphotransferase (neo); thymidine kinase (TK); bacterial artificial chromosome (BAC); fast protein liquid chromatography (FPLC); lysophosphatidyl choline (Lyso-PC); phosphatidyl ethanolamine (PE); phosphatidyl choline (PC). phospholipid transfer protein (PLTP). Intense study over the last few years has focused on the HDL particle in the context of reverse cholesterol transport and antioxidant properties, providing significant insights into the mechanism of the antiatherogenic properties of $\operatorname{HDL}(7,8)$. Despite significant insights gained through these studies, there continue to be large gaps in our understanding of HDL metabolism. Further study is required to better understand how HDL particles may be manipulated with regard to both level and composition to affect the atherosclerotic disease process through therapeutic intervention.

Serum HDL cholesterol levels are regulated in part by members of the lipase enzyme family. The lipases have highly conserved structural domains, and these enzymes function to metabolize triglycerides and phospholipids. Two members of this family, HL and lipoprotein lipase (LPL), are important in the processing of lipids carried within lipoproteins and probably also in the uptake of lipoprotein particles into cells (9). Although synthesized in nonendothelial cells, these two secreted enzymes translocate to the surface of endothelial cells, where they carry out their metabolic function. Both HL and LPL have been implicated in atherosclerotic vascular disease (10-15). Recently, the lipase gene family was extended through the characterization of endothelial lipase (protein, EL; gene, LIPG; NCBI Locuslink 9388) $(16,17)$. LIPG has been shown to be expressed by vascular cells in vivo and to be highly regulated in vitro in endothelial cells by cytokines and biophysical forces (18). This novel lipase is also expressed by a wide range of nonendothe- 
lial cells in a variety of tissues, including the placenta, liver, lung, ovary, thyroid gland, and testis $(16,17)$. EL has been shown to have phospholipase activity and relatively little triglyceride lipase activity $(16,19)$. One previous study has demonstrated that high-level overexpression of EL in the liver by adenovirus-mediated gene transfer results in a significant decrease in HDL cholesterol and apoA-I, suggesting that EL could be a physiological regulator of HDL metabolism (17).

In this study, we have sought to further explore a potential role for EL in HDL cholesterol metabolism. Toward this end, we have generated murine genetic models with altered levels of EL expression. Decreased LIPG expression was accomplished by gene targeting and development of mice functionally lacking one or two LIPG alleles, and increased expression was accomplished by the development of transgenic animals carrying copies of the human LIPG locus. Study of lipid levels in these animals revealed a strong inverse relationship between EL expression and HDL cholesterol levels. The genetic models revealed a strong correlation between phospholipase activity and EL expression and demonstrated that, in the mouse, EL is responsible for the bulk of heparin-releasable phospholipase activity. A modest correlation was found between EL expression and triglyceride levels. These studies thus establish a physiological role for EL in lipoprotein metabolism.

\section{Methods}

Generation of EL genetic models. A mouse LIPG cDNA probe (EcoRI-PstI fragment) was employed for screening a $\lambda$ phage $129 /$ SvJ genomic library (Stratagene, La Jolla, California, USA), providing two overlapping clones of 15 and $13 \mathrm{~kb}$. These phage clones were restriction mapped, and the exon/intron structure was partially determined by mapping and nucleotide sequence analysis. The replacement targeting vector was constructed in the pKO Scrambler NTKV-1901 vector (Stratagene) using a 4.6-kb HindIII-XbaI fragment for the $5^{\prime}$ arm and a 3.6-kb KpnI-XhoI fragment for the 3' arm (Figure 1). The homology regions used and their organization in the vector was designed to replace exon 1 of LIPG with a neomycin phosphotransferase (neo) cassette. Also, a cassette for herpes simplex virus thymidine kinase (TK) was provided outside the region of homology to allow negative selection.

TL-1 129 ES cells (Brigid Hogan, Vanderbilt University, Nashville, Tennessee, USA) were grown in $\mathrm{DMEM} / 10 \%$ FCS containing $1000 \mathrm{U} / \mathrm{ml}$ leukemia inhibitory factor (GIBCO BRL/Invitrogen, Carlsbad, California, USA). A total of $20 \times 10^{6}$ cells, at passage 10 , were electroporated with $50 \mu \mathrm{g}$ of linearized targeting vector with a single pulse at $800 \mathrm{~V}$ and $3 \mu \mathrm{F}$. The next day, selection was started with $300 \mu \mathrm{g} / \mathrm{ml} \mathrm{G} 418$, followed 2 days later with the addition of gancyclovir at a concentration of $2 \mu \mathrm{mol} / \mathrm{L}$. After 10-14 days, double resistant clones were picked and expanded, and genomic DNA was isolated and evaluated by Southern blot with $5^{\prime}$ and $3^{\prime}$ probes outside the homology domains (Figure 1). One of the correctly targeted ES cell clones was injected into $\mathrm{C} 57 \mathrm{Bl} / 6$ blastocysts to generate chimeric animals. A single chimeric male was bred to C57Bl/6 females (Jackson Labs, Bar Harbor, Maine, USA) to obtain heterozygous pups. Male heterozygous pups were bred with $\mathrm{C} 57 \mathrm{Bl} / 6$ female mice eight times before homozygous animals were generated. Littermates served as controls for all lines studied. Genotyping of knockout animals was performed with DNA isolated from tail tissue, which was digested with EcoRI and separated on $0.65 \%$ agarose gels. After transfer to nylon membranes, blots were hybridized with $\left[{ }^{32} \mathrm{P}\right] 2^{\prime}-$ deoxycytidine $5^{\prime}$-triphosphate-labeled probes synthesized using 5'(EcoRI-BamHI) or 3' (HindIII-BglII) fragments by random priming (Figure 1). Genotypes of mice were verified by detection of the fragments that differ in size between the wild-type and targeted locus.

For the generation of transgenic mice carrying the human LIPG locus, PCR primers based on the cDNA sequence, $5^{\prime}$-gtaaaatgtgagcatgagcgag- $3^{\prime}$ and $5^{\prime}$-ttcttggcattgtagccaatg-3', were used to screen an arrayed bacterial artificial chromosome (BAC) library (20). The BAC spans from nucleotide $47,198,300$ to nucleotide $47,395,100$ in the June 2002 version of the human whole-genome assembly. This matches well with the estimated size of the BAC, $196 \mathrm{~kb}$. The LIPG gene is located $5^{\prime}$ to $3^{\prime}$ from nucleotide $47,367,696$ to nucleotide $47,247,058$, which indicates that this BAC contains about $27.4 \mathrm{~kb}$ of $5^{\prime}$ and $48.7 \mathrm{~kb}$ of $3^{\prime}$ flanking regions. According to the whole-genome annotation, there is no other gene except a ribosomal protein gene, $R P L 17$, located in an intron of the LIPG gene in this BAC. BAC DNA isolated with standard alkaline lysis techniques was injected into the pronuclei of C57Bl/ 6 fertilized eggs. Transgenic animals (hereafter referred to as hLIPGTg) were identified by Southern blot of BglIIdigested tail DNA with a human LIPG cDNA probe.

$R N A$ and protein expression studies. Mouse tissues were homogenized in TRIZOL (Invitrogen), and the aqueous phase was extracted after adding chloroform. After precipitating the RNA with isopropanol, the RNA pellets were dissolved in the RLT lysis buffer with $\beta$-mercaptoethanol and cleaned with RNeasy columns (Qiagen, Valencia, California, USA).

For Northern blot analysis, $20 \mu \mathrm{g}$ of total RNA was separated on formaldehyde-denaturing $1.0 \%$ agarose gels and transferred to nylon membranes. Membranes were then hybridized to a mouse LIPG (1.5-kb) cDNA probe radiolabeled with $\left[{ }^{32} \mathrm{P}\right] 2^{\prime}$-deoxycytidine $5^{\prime}$-triphosphate by random priming. Blots were hybridized at $42^{\circ} \mathrm{C}$ for $16-24$ hours in the presence of $48 \%$ formamide and $10 \%$ dextran sulfate and then washed at high-stringency conditions at $65^{\circ} \mathrm{C}$ in the presence of $0.2 \times \mathrm{SSC}$ buffer and $0.5 \%$ SDS. Visualization was achieved by exposure to Kodak Biomax MS film (Eastman Kodak, Rochester, New York, USA).

For RNase protection assays, a SacI-NdeI fragment of human $L I P G$ was cloned into the PSP72 vector, and an 


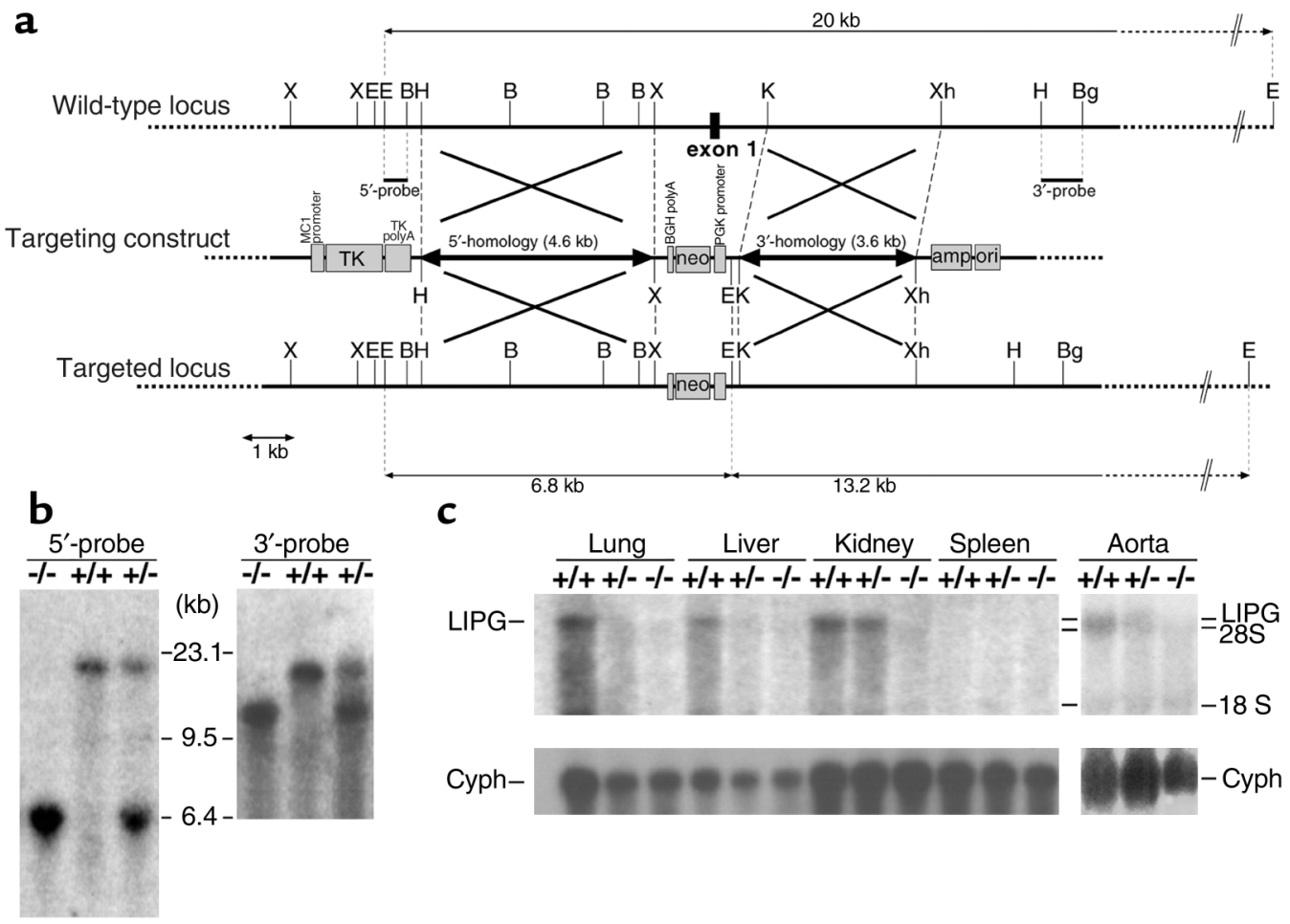

\begin{abstract}
Figure 1
Targeting of the mouse endothelial lipase gene. (a) The wild-type locus of mouse LIPG (top), the targeting construct (middle), and the targeted locus (bottom). Exon 1 was replaced with the neomycin phosphotransferase gene (neo), and recombination was detected by Southern blot analysis using the probes indicated. X, Xbal; B, BamHI; K, Kpnl; Bg, Bg/ll; H, HindIII; E, EcoRI; Xh, Xhol. (b) Both probes gave a 20-kb wildtype band on Southern blotting, whereas the targeted recombinants showed a 6.8-kb band with the 5'probe and a 13.2-kb band with the 3'probe. Targeted stem cells were characterized by the presence of both DNA fragments. (c) Northern blot analysis showed LIPG expression in mouse tissues. A full-length mouse LIPG cDNA was used as a probe. Cyph, cyclophilin.
\end{abstract}

EcoRI-EcoRI fragment of mouse LIPG was cloned into the pBluescript $\mathrm{SK}(+)$ vector. Both plasmids were linearized with XhoI. $\left.{ }^{32} \mathrm{P}\right] \mathrm{UTP}-\mathrm{labeled}$ antisense riboprobe was synthesized with T7 RNA polymerase, and an RNase protection assay was performed as described previously (21). Protection of human LIPG transcripts resulted in a labeled fragment of 694 nucleotides, protection of mouse LIPG transcripts resulted in a labeled fragment of 610 nucleotides, and protection of $\beta$-actin transcripts resulted in a labeled fragment of 250 nucleotides.

For Western blot analysis, mouse tissues from LIPG transgenic mice were homogenized in lysis buffer (10 $\mathrm{mmol} / \mathrm{l}$ Tris [pH 7.4], $150 \mathrm{mmol} / \mathrm{l} \mathrm{NaCl}, 2 \mathrm{mmol} / \mathrm{l}$ $\mathrm{CaCl}_{2}, 1 \% \mathrm{NP}-40,1 \%$ Triton X-100, $1 \mathrm{mmol} / 1$ PMSF, $40 \mathrm{U} / \mathrm{ml}$ aprotinin, $15 \mu \mathrm{g} / \mathrm{ml}$ leupeptin). Aliquots (50 $\mu \mathrm{g}$ ) of protein solution were boiled with Laemmli buffer and subjected to SDS-PAGE. The gel was transferred to polyvinylidene fluoride membrane and blocked with 5\% nonfat dry milk. Anti-human EL antibody was used as the primary antibody. The primary antibody employed was an anti-human polyclonal EL antibody raised in rabbit against a synthetic peptide encoding residues $422-445$ of processed EL, SWYNLWKEFRSYLSQPRNPGRELN. The membrane was incubated with anti-EL antibody (1:1000) and then anti-rabbit IgG-HRP (Amersham-Pharmacia, Pis- cataway, New Jersey, USA). The signal was detected by chemiluminescent reaction.

Immunobistochemistry. Wild type and $\mathrm{h} L I P G \mathrm{Tg}$ mice were anesthetized by administration of pentobarbital and perfused with $4 \%$ paraformaldehyde/PBS at $10 \mathrm{~mm}$ $\mathrm{Hg}$ for lungs and at $130 \mathrm{~mm} \mathrm{Hg}$ for other tissues. Tissues were excised and further fixed in $4 \%$ paraformaldehyde/PBS, and then paraffin-embedded sections were made by use of standard methods. The sections were incubated with $3 \% \mathrm{H}_{2} \mathrm{O}_{2}$ /methanol for 20 minutes to eliminate endogenous peroxidase, washed with PBS, blocked with carrier protein (DAKO LSAB kit, DAKO A/S, Glostrup, Denmark) for 1 hour at room temperature, and then incubated with primary antibody overnight at $4^{\circ} \mathrm{C}$. The primary antibody was an antihuman monoclonal EL antibody raised in mouse against a synthetic peptide encoding residues 18-32 of processed EL, AGSPVPFGPEGRLE. This antibody does not crossreact with murine EL. After washing with PBS, sections were incubated with biotinylated goat anti-mouse IgG (1:300), washed with PBS, and then incubated with streptavidin-horseradish peroxidase conjugate (DAKO A/S). After color development with $0.03 \%$ diaminobenzidine, the sections were counterstained with Mayer's hematoxylin for 120 seconds. As a negative control, the primary antibody was replaced with mouse nonspecific Ig. 
Analysis of plasma lipids. Following an overnight fast, $200-400 \mu \mathrm{l}$ of blood was collected by retro-orbital bleeding into tubes containing $0.3 \mathrm{mg}$ of EDTA. Plasma was collected by centrifugation at $8000 \mathrm{~g}$ for 10 minutes. Total cholesterol, HDL and LDL cholesterol, and triglycerides were measured with standard enzymatic assays by Anylitics Inc. (Gaithersburg, Maryland, USA), using the HITACHI 717 Chemical Analyzer (Rankin Biomedical Corp., Clarkston, Michigan, USA). Also, total plasma cholesterol levels were determined by the cholesterol oxidase method, and total plasma triglyceride levels were determined by the glycerindepletion method, using commercially available kits (Wako Pure Chemicals, Osaka, Japan).

Separation of plasma lipoproteins by fast protein liquid chromatography (FPLC) was performed using two Suprose 6 columns (Amersham-Pharmacia) as described previously (22). Briefly, $100 \mu \mathrm{l}$ of mouse plasma samples were applied to FPLC, and 37 fractions $(1 \mathrm{ml})$ were collected and analyzed for total cholesterol and triglycerides. Total cholesterol and triglycerides were assayed using enzymatic kits from Sigma-Aldrich (St. Louis, Missouri, USA). Some fractions were further analyzed by SDS-PAGE followed by Coomassie brilliant blue staining of the separated proteins. For Western blotting, $100 \mu$ l of FPLC fractions containing HDL were used for 12\% SDS-PAGE and transferred to nitrocellulose membrane (Bio-Rad, Hercules, California, USA). The membrane was incubated with a 1:500 dilution of mouse human apoA-I antibodies (ICN Biochemicals, Aurora, Ohio, USA) followed by incubation with secondary antibody conjugated with HRP (1:4000). The apoA-I was then visualized using an ECL technique (Amersham-Pharmacia). The numbers of animals used for FPLC analysis were as follows: 8 for $\mathrm{LIPG}^{-/-}, 7$ for wild type, and 5 for $\mathrm{h} L I P G T g$.

For ultracentrifugation apolipoprotein analysis, plasma samples were collected by heart puncture in a syringe containing EDTA. Two separate pooled plasma samples were analyzed, with five animals included in each pooled sample. Lipoproteins were fractionated by density-gradient ultracentrifugation as described by Havel et al. (23), and the HDL fraction corresponding to $d=1.063-1.21$ was used for analyses. Protein was quantitated by the Lowry biochemical method and evaluated by SDS-polyacrylamide gel electrophoresis followed by Coomassie brilliant blue staining of the gels. Cholesterol and phospholipid were measured with kits from Sigma-Aldrich and Wako Pure Chemicals, respectively, as described above. TLC was performed as follows. A total of $35 \mu \mathrm{g}$ of the HDL fraction was extracted using a method described by Bligh and Dyer (24). The chloroform phase was dried under nitrogen, and the lipid was redissolved with $50 \mu \mathrm{l}$ of a chloroform/methanol (1:1) solution and applied on a TLC plate. Purified lysophosphatidyl choline (Lyso-PC), phosphatidyl ethanolamine (PE), and phosphatidyl choline (PC) (Sigma-Aldrich) were used as standards. The lipids were separated using a solvent mixture of methanol, chloroform, and water (130:70:12) and visualized by staining with iodine vapor.

Biochemical determination of phospholipid concentrations in mouse plasma and HDL fractions was performed using the Phospholipid-TestWako kit (Wako Pure Chemicals). Phospholipase activity was quantitated as follows. Mouse plasma was collected into EDTAcontaining tubes before and 10 minutes after intravenous injection of heparin $(150 \mathrm{U} / \mathrm{kg})$. PC emulsion was made by combining $\left[{ }^{14} \mathrm{C}\right]$ dipalmitoyl PC $(1 \mu \mathrm{Ci})$ (New England Nuclear, Boston, Massachusetts, USA) and lecithin followed by evaporation under nitrogen. One milliliter of $100 \mathrm{mM}$ Tris- $\mathrm{HCl}(\mathrm{pH}$ 7.4) containing $1 \%$ Triton X-100, $5 \mathrm{mM} \mathrm{CaCl}_{2}, 200 \mathrm{mM} \mathrm{NaCl}$, and $0.1 \%$ BSA was added and vortexed. Sixty microliters of emulsion were then added to $100 \mu \mathrm{l}$ of plasma samples and incubated for 2 hours at $37^{\circ} \mathrm{C}$. Labeled fatty acids were separated by the liquid-liquid separation as described previously (25). Briefly, the reaction was terminated by the addition of $3 \mathrm{ml}$ of Dole's reagent (isopropanol/ n-heptane $/ 0.5 \mathrm{M} \mathrm{H}_{2} \mathrm{SO}_{4}, 78: 20: 2$ [vol/vol]). The tubes were vortexed vigorously, and distilled water $(1.6 \mathrm{ml})$ and heptane $(1.8 \mathrm{ml})$ were added and vortexed. The emulsion was separated by centrifugation at $3000 \mathrm{~g}$ for 15 minutes. One and a half milliliters of the upper phase was removed to another tube containing $100 \mathrm{mg}$ of silica gel. After vortexing for 20 seconds, the silica gel was precipitated by centrifugation at $100 \mathrm{~g}$ for 10 minutes. Heptane $(1 \mathrm{ml})$ was removed for radioactivity counting in a scintillation counter. Four animals were included in each of the different genotype groups for this analysis.

Statistical analysis. Data are shown as means \pm SEM. The $t$ test for unequal variances or the Student's $t$ test (two tailed) was used to determine the statistical significance of differences between the groups of animals. Differences with a $P$ value less than 0.05 were considered significant.

\section{Results}

Production of EL genetic mouse models. Mapping and nucleotide sequence analysis revealed a $5^{\prime}$-most exon that contained $5^{\prime}$ untranslated sequence, the initiating methionine and signal sequence of the murine LIPG gene, corresponding to published cDNA sequence. The organization of this exon (hereafter referred to as exon 1) (Figure 1a) is similar to what is seen in the human LIPG locus. However, in humans there is an upstream alternative first exon that encodes EL isoforms that do not encode a signal sequence ( $T$. Ishida et al., manuscript submitted for publication). Mapping and sequence analysis failed to reveal an orthologous region in the mouse genome, so the single identified $5^{\prime}$ exon 1 was targeted to be replaced with the neomycin resistance gene. Southern blot analysis was performed with both $5^{\prime}$ and $3^{\prime}$ probes to verify substitution of the targeted region by homologous recombination (Figure $1 \mathrm{~b}$ ). To demonstrate that the LIPG gene was inactivated in $L I P G^{-/-}$mice, several tissue RNAs were prepared and analyzed by Northern blotting using a full-length mouse LIPG cDNA probe. As shown in Figure 1c, 
although wild-type mice expressed a large amount of LIPG mRNA in lung, kidney, aorta, liver, and heart, $L I P G^{-/-}$mice were deficient in this mRNA in these organs. $L I P G^{-1-}$ mice were viable and fertile and did not exhibit overt defects. Various organs from $L I P G^{-/-}$mice were further examined histologically, and no abnormalities were detected by light microscopy (data not shown). There was no difference in body weight between wild-type and $\mathrm{LIPG}^{-/-}$mice (data not shown).

BAC DNA microinjection produced mice that had integrated the human transgene, as determined by Southern blot using a human LIPG cDNA probe (Figure 2a). Expression of hLIPGTg in these animals was evaluated by RNase protection assay using radiolabeled human LIPG riboprobes that did not cross-react with the native mouse LIPG. The hLIPGTg animals were found to have expression of the human LIPG transgene, with an RNase protection assay detecting message in brain, aorta, heart, lung, kidney, and spleen (Figure 2b). Overall, these data indicated that expression of the human BAC produced a 3.2-fold greater level of human LIPG mRNA than the endogenous murine gene. By Western blotting of murine tissue extracts, hLIPGTg produced protein levels of human EL approximately 1.9 -fold greater than endogenous murine EL (data not shown). Tissue localization of human EL was conducted by immunohistochemistry (Figure 2c). These data confirmed that human LIPG mRNA documented by RNase protection resulted in protein expression in aorta, kidney, lung, and spleen. Human EL was found to colocalize with endothelial cells associated with the aorta and large vessels in the kidney and lung as well as the microcirculation in lung and spleen (arrows, Figure 2c). Although LIPG has been shown to be expressed in the liver in humans, and in mice as shown here, there was no hepatic expression of the transgene. This result probably reflects the existence of distant hepatic regulatory elements of the LIPG gene that were not found on this BAC. The $\mathrm{h} L I P G T g$ mice were viable and fertile and did not exhibit overt defects. Various organs from hLIPGTg mice showed no histological abnormalities when evaluated by light microscopy (data not shown). There was no difference in body weight between wild-type and hLIPGTg mice (data not shown).

Plasma lipid and lipoprotein profiles. Fasting plasma lipid levels of wild-type, $L I P G^{+/-}, L I P G^{-/}$, and $\mathrm{h} L I P G T g$ mice are listed in Table 1 . In both male and female $L I P G^{-/-}$ mice, plasma levels of total cholesterol were significantly increased, with a $69 \%$ increase in male homozygous knockout mice as compared with wild-type littermate controls. This was primarily due to an increase in

$$
\mathbf{a}
$$
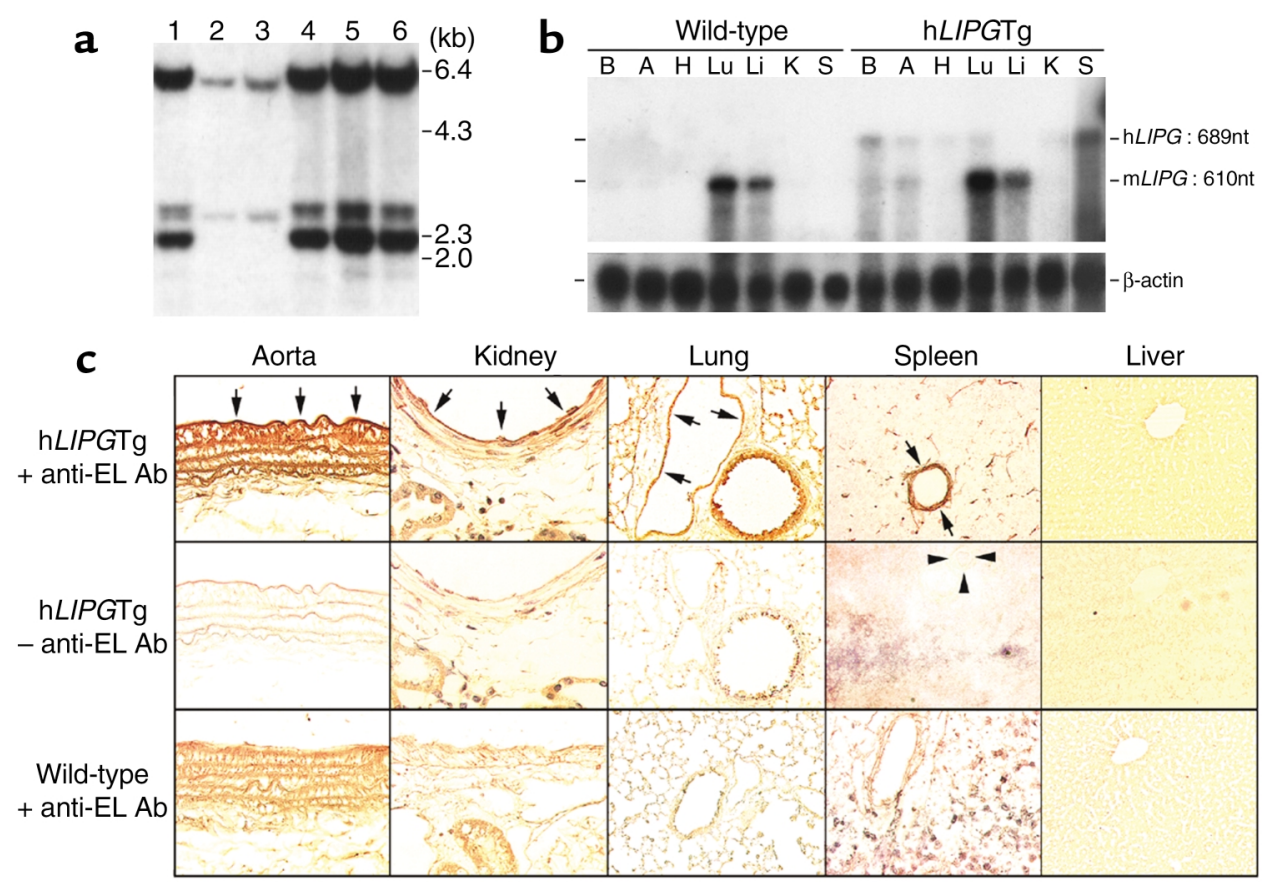

\section{Figure 2}

Generation of human endothelial lipase transgenic mice. (a) Genotyping of LIPG transgenic mice. Southern blot analysis was employed to detect the human LIPG transgene. Lanes 2 and 3 show wild-type mice; lanes 1, 4, and 6, heterozygous transgenic mice; and lane 5, homozygous transgenic mice. (b) RNase protection with hLIPGTg mouse RNAs. Human LIPG-specific signals were detected in the brain, aorta, heart, lung, kidney, and spleen. B, brain; A, aorta; H, heart; Lu, lung; Li, liver; K, kidney; S, spleen. (c) Immunohistochemical evaluation of human EL expression in transgenic mice. Tissues harvested from hLIPGTg mice were studied with an anti-human EL monoclonal antibody raised against a synthetic peptide. Human EL was primarily associated with the endothelium of the aorta and large vessels in the kidney, lung, and spleen as well as the microcirculation in the spleen and lung (arrows). Arrowheads in the spleen indicate the central artery, which does not stain when the primary antibody is omitted. No expression was detected in the liver. Original magnification: aorta, $\times 400 ;$ lung and liver, $\times 200$; kidney and spleen, $\times 630$. 
Table 1

Lipid and lipoprotein profile in fasted EL knockout and transgenic mice on normal chow

\begin{tabular}{|c|c|c|c|c|c|}
\hline Male & Chol & TG & HDL-C & LDL-C & PL \\
\hline $\begin{array}{l}\mathrm{LIPG}^{-/-} \\
P \text { value } \\
n\end{array}$ & $\begin{array}{c}162.0 \pm 7.3 \\
<0.001 \\
11\end{array}$ & $\begin{array}{c}85.6 \pm 6.0 \\
0.83 \\
11\end{array}$ & $\begin{array}{c}119.2 \pm 5.2 \\
<0.001 \\
11\end{array}$ & $\begin{array}{c}11.8 \pm 0.6 \\
<0.001 \\
6\end{array}$ & $\begin{array}{c}218.4 \pm 7.6 \\
0.0017 \\
8\end{array}$ \\
\hline $\begin{array}{l}\mathrm{LIPG}^{+/-} \\
P \text { value } \\
n\end{array}$ & $\begin{array}{c}124.4 \pm 5.9 \\
0.00119 \\
8\end{array}$ & $\begin{array}{c}97.2 \pm 9.2 \\
0.2856 \\
8\end{array}$ & $\begin{array}{c}95.1 \pm 4.1 \\
0.00265 \\
8\end{array}$ & $\begin{array}{c}6.6 \pm 0.4 \\
0.665 \\
5\end{array}$ & $\begin{array}{c}188.8 \pm 6.4 \\
0.0177 \\
8\end{array}$ \\
\hline $\begin{array}{l}\text { Wild-type } \\
n\end{array}$ & $\begin{array}{c}95.6 \pm 4.0 \\
10\end{array}$ & $\begin{array}{c}83.3 \pm 8.8 \\
10\end{array}$ & $\begin{array}{c}76.0 \pm 3.4 \\
10\end{array}$ & $\begin{array}{c}6.2 \pm 0.7 \\
6\end{array}$ & $\begin{array}{c}142.8 \pm 7.2 \\
5\end{array}$ \\
\hline $\begin{array}{l}\text { hLIPGTg } \\
P \text { value } \\
n\end{array}$ & $\begin{array}{c}78.9 \pm 4.0 \\
0.0095 \\
8\end{array}$ & $\begin{array}{c}62.1 \pm 5.7 \\
0.0618 \\
8\end{array}$ & $\begin{array}{c}61.2 \pm 3.3 \\
0.00687 \\
8\end{array}$ & $\begin{array}{c}4.5 \pm 0.9 \\
0.214 \\
5\end{array}$ & $\begin{array}{c}112.0 \pm 7.5 \\
0.098 \\
5\end{array}$ \\
\hline \multicolumn{6}{|l|}{ Female } \\
\hline $\begin{array}{l}L_{L P G^{-/-}} \\
P \text { value } \\
n\end{array}$ & $\begin{array}{c}99.5 \pm 5.6 \\
0.0061 \\
11\end{array}$ & $\begin{array}{c}86.0 \pm 8.7 \\
0.055 \\
11\end{array}$ & $\begin{array}{c}75.0 \pm 6.1 \\
0.0045 \\
11\end{array}$ & $\begin{array}{c}8.3 \pm 0.7 \\
0.905 \\
6\end{array}$ & $\begin{array}{c}154.6 \pm 11.2 \\
0.0921 \\
5\end{array}$ \\
\hline $\begin{array}{l}\mathrm{LIPG}^{+/-} \\
P \text { value } \\
n\end{array}$ & $\begin{array}{c}93.0 \pm 12.8 \\
0.0467 \\
9\end{array}$ & $\begin{array}{c}89.4 \pm 13.1 \\
0.0158 \\
9\end{array}$ & $\begin{array}{c}69.8 \pm 9.7 \\
0.0083 \\
9\end{array}$ & $\begin{array}{c}6.0 \pm 1.1 \\
0.0674 \\
5\end{array}$ & $\begin{array}{c}133.8 \pm 34.2 \\
0.0058 \\
5\end{array}$ \\
\hline $\begin{array}{l}\text { Wild-type } \\
n\end{array}$ & $\begin{array}{c}72.0 \pm 0.8 \\
8\end{array}$ & $\begin{array}{c}66.8 \pm 2.7 \\
8\end{array}$ & $\begin{array}{c}52.8 \pm 2.0 \\
8\end{array}$ & $\begin{array}{c}8.4 \pm 0.7 \\
5\end{array}$ & $\frac{102.1 \pm 10.6}{5}$ \\
\hline $\begin{array}{l}\text { hLIPGTg } \\
P \text { value } \\
n\end{array}$ & $\begin{array}{c}64.7 \pm 3.7 \\
0.0021 \\
11\end{array}$ & $\begin{array}{c}60.8 \pm 6.8 \\
0.426 \\
11\end{array}$ & $\begin{array}{c}45.3 \pm 2.2 \\
0.0224 \\
10\end{array}$ & $\begin{array}{c}8.1 \pm 0.7 \\
0.798 \\
6\end{array}$ & $\begin{array}{c}82.1 \pm 3.4 \\
0.033 \\
5\end{array}$ \\
\hline
\end{tabular}

Data are shown as means $\pm \mathrm{SEM}(\mathrm{mg} / \mathrm{dl})$. $P$ values represent comparison with the wild-type group. Chol, cholesterol; TG, triglyceride; HDL-C, HDL cholesterol; LDL-C, LDL cholesterol; PL, phospholipids. of wild-type controls. Thus, phospholipid levels varied with respect to genotype in a pattern similar to that observed for cholesterol level.

Plasma lipoprotein profiles were determined by FPLC (Figure 3). The $L I P G^{-/-}$mice had a significant $21 \%$ increase in HDL cholesterol in fractions 28 and 29. The hLIPGTg mice showed a significant $78 \%$ decrease in HDL cholesterol in fraction 29 and a significant $80 \%$ decrease in fraction 30 . These cholesterol levels obtained on HDL peak fractions from FPLC were different from those obtained by biochemical measurements on whole plasma (Table 1 and Figure 3). The increase in HDL cholesterol levels for the knockout mice was smaller when measured on the FPLC fractions, and the HDL cholesterol decrease observed in the transgenic mice was greater when determined on FPLC fractions.

By FPLC, the $L I P G^{-/-}$mice had a minimal increase in cholesterol in LDL-containing fractions, and hLIPGTg mice had a corresponding decrease, although neither of these was significant.

HDL cholesterol levels, which were increased by $57 \%$ in $L I P G^{-/-}$and $25 \%$ in $L I P G^{+/-}$male mice, with female mice showing similar levels of increase as wild-type controls. Conversely, hLIPGTg male mice showed total and HDL cholesterol levels to be decreased by $17 \%$ and $19 \%$, respectively. It is interesting to note that LDL cholesterol levels were increased by $90 \%$ in male $\mathrm{LIPG}^{-/-}$mice as compared with wild-type mice. The significance of this finding is unclear, however, since there was no significant change in LDL cholesterol levels in any of the other genotype groups for either male or female mice.

Measurement of triglyceride levels in the genetic mouse models indicated that EL may have a modest in vivo role in triglyceride metabolism (Table 1). In female $L I P G^{+/-}$animals, a significantly greater level of triglycerides was measured than in wild-type controls. Although triglyceride levels were not statistically different among the other genetic models, for female $L I P G^{-/-}$animals there was an increase that approached significance, and in the male hLIPGTg mice there was a decreased mean value that approached significance.

Plasma phospholipid levels tended to be higher in animals missing functional endogenous LIPG alleles and lower in overexpressing animals (Table 1). Both male and female $\mathrm{LIPG}^{-1}$ animals showed a significant increase in plasma phospholipid levels of over 50\%, whereas $L I P G^{+/-}$animals showed a significant increase in phospholipid levels of over $30 \%$. The overexpressing $\mathrm{h}$ LIPGTg female mice had a significant, $19 \%$ lower phospholipid level, and the male hLIPGTg mice had a $21 \%$ lower level that was not significantly different from that
Apolipoprotein analysis. Lipoprotein fractions isolated by FPLC were evaluated by polyacrylamide gel electrophoresis and Western blotting, to determine if the cholesterol changes associated with altered EL expression levels reflected alterations in the apoprotein components of the lipoprotein particles. Electrophoresis on a $15 \%$ gel of the peak HDL-containing fractions revealed that there were differences in the intensity of the Coomassie-stained bands representing apoA-I in

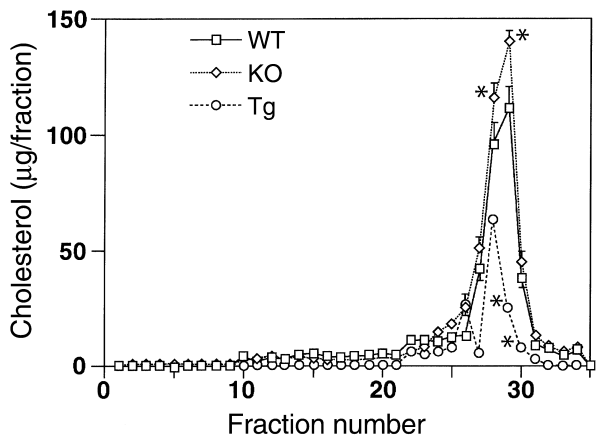

\section{Figure 3}

Cholesterol levels in lipoprotein fractions obtained by FPLC. Determination of cholesterol concentration in serum fractions obtained by FPLC revealed a correlation between LIPG genotype and HDL cholesterol levels. HDL cholesterol levels for $L I P G^{-1-}$ mice were significantly greater than for wild-type mice in fractions 28 and 29 $(P<0.05)$. HDL cholesterol levels were lower in LIPG transgenic animals for fractions 29 and $30(P<0.05)$ than in wild-type mice. KO, LIPG $^{-1-} ; \mathrm{Tg}, \mathrm{hLIPGTg} .{ }^{*} P<0.05$ vs. WT. 


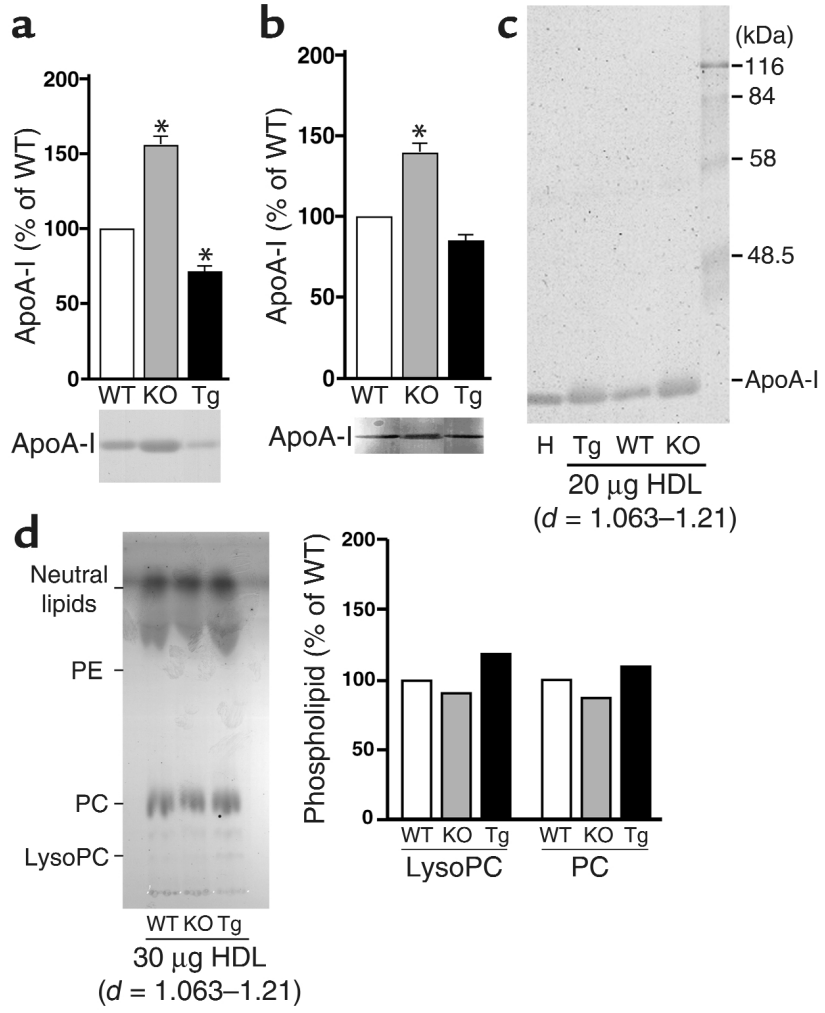

Figure 4

HDL lipoprotein particle analysis. (a) SDS-PAGE analysis of apoA-I in the HDL peak fractions isolated by FPLC. One hundred microliters of the HDL peak fraction from FPLC were evaluated by $15 \%$ SDS-PAGE. Gels were stained and quantitated by densitometry. (b) Western blot analysis of apoA-I in HDL peak fraction from FPLC. Images were quantitated by densitometry. (c) HDL isolated by density-gradient ultracentrifugation $(d=1.063-1.21)$ was evaluated by $12 \%$ SDSPAGE, with $20 \mu \mathrm{g}$ of protein loaded per lane. Lane 1 is human HDL standard, and lane 5 is a molecular weight marker. $\mathrm{H}$, human $\mathrm{HDL}$ standard. (d) HDL isolated by density-gradient ultracentrifugation $(d=1.063-1.21)$ was evaluated by TLC. Equal amounts of HDL, as determined by protein quantitation, were loaded. $\mathrm{KO}, \mathrm{LIPG}^{-1-} ; \mathrm{Tg}$, hLIPGTg. ${ }^{*} P<0.05$ compared to WT.

the different genetic models (Figure 4a). Replication of this experiment and quantitation by densitometric scanning of the gels revealed a significant $52 \%$ increase in the apoA-I levels in the homozygous knockout animals and a significant $26 \%$ decrease in transgenic animals. Similar experiments were conducted with transfer of the electrophoresed protein to membranes that were probed with an antibody to apoA-I (Figure 4b). Quantitation of these results detected a significant $40 \%$ increase in apoA-I in the homozygous knockout animals and a $17 \%$ decrease in transgenic animals that was not significant. To further investigate the apolipoprotein content of the HDL fractions from FPLC, SDSPAGE was performed on the HDL peak fraction with a $3-15 \%$ gradient gel. This gel revealed the presence of apoB in these fractions, suggesting some contamination of HDL with small LDL (data not shown). Such contamination could contribute to the observed dif- ferences in HDL cholesterol levels measured by biochemical techniques versus FPLC (Table 1 and Figure 3). Similar analysis of LDL fractions from FPLC revealed no evidence of apoA-I.

To further define the composition of HDL particles in the genetic models, ultracentrifugation fractionation of lipoprotein particles was performed. When equal amounts of protein from the HDL fraction (density, $1.063-1.21 \mathrm{~g} / \mathrm{ml}$ ) were analyzed by SDS-PAGE, a single band with equal density corresponding to apoA-I was obtained for the different genetic models (Figure 4c). Analysis of the same ultracentrifugation density fraction by TLC was performed to evaluate the phospholipid makeup of the HDL particles among the different mouse models (Figure 4d). When equal amounts of HDL were evaluated, the results were not statistically different among the wild-type, homozygous knockout, and transgenic lines. In particular, no accumulation of lysophosphatidyl choline was seen in the knockout or transgenic lines.

The ultracentrifugal HDL fraction was analyzed for protein, cholesterol, and phospholipid content (Table 2). These data indicated that the composition of HDL particles in the knockout and transgenic lines was similar to that of the wild-type animals.

Phospholipase activity. Phospholipase activity was compared among $L I P G^{-1-}, \mathrm{h} L I P G T g$, and wild-type animals before and after heparin injection (Figure 5). Before heparin injection, phospholipase activity was not statistically different among the different lines (wild-type mice, $519 \pm 22 \mathrm{nmol} \mathrm{FFA} / \mathrm{ml} / \mathrm{hr}$; knockout mice, $521 \pm 5$ $\mathrm{nmol} \mathrm{FFA} / \mathrm{ml} / \mathrm{hr}$; transgenic mice, $510.5 \pm 20.23 \mathrm{nmol}$ $\mathrm{FFA} / \mathrm{ml} / \mathrm{hr}$ ). After heparin injection, there was a $32 \%$ increase in phospholipase activity in the wild-type animals. A significantly greater $45 \%$ increase in postheparin phospholipase activity was observed for transgenic animals. For $L I P G^{-/-}$mice, there was a $12 \%$ postheparin increase in phospholipase activity, which was significantly less than that observed in wild-type mice. These data indicate that over $50 \%$ of heparin-releasable phospholipase activity is associated with EL.

\section{Discussion}

LIPG was originally cloned by two groups looking for differentially regulated genes in two very different in vitro systems. One group differentiated THP-1 cells and identified upregulated genes by differential dis-

\section{Table 2}

HDL lipoprotein particle composition

\begin{tabular}{lccc}
\hline & Protein (\%) & Cholesterol (\%) & Phospholipid (\%) \\
Wild-type & 48.0 & 6.8 & 43.0 \\
LIPG & 43.5 & 7.1 & 50.0 \\
hLIPGTg & 44.0 & 6.4 & 51.0
\end{tabular}

The averages of measurements for two groups of five pooled mouse plasma samples are shown. Data are expressed as mean percents by weight. Cholesterol represents the total cholesterol of the particle; free cholesterol and cholesterol ester were not measured separately. 


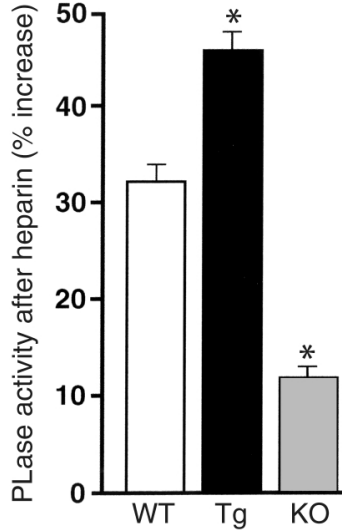

\begin{abstract}
Figure 5
Phospholipase activity in genetic models. Phospholipase activity in different genetic lines was compared between pre- and postheparin injection samples. A significantly greater level of postheparin phospholipase activity was observed in the transgenic lines $\left({ }^{*} P<0.05\right)$, and a significantly lower level of postheparin augmentation of phospholipase activity was found in the knockout animals $\left({ }^{*} P<0.05\right)$ as compared to the wild-type controls. The preheparin levels of plasma phospholipase activity were as follows (mean \pm SEM): wild-type animals, $519 \pm 22.3 \mathrm{nmol} \mathrm{FFA} / \mathrm{ml} / \mathrm{hr}$; knockout animals, $521 \pm 5.4$ $\mathrm{nmol} \mathrm{FFA} / \mathrm{ml} / \mathrm{hr}$; and transgenic animals, $510.5 \pm 20.2 \mathrm{nmol}$ $\mathrm{FFA} / \mathrm{ml} / \mathrm{hr}$. PLase, phospholipase activity; Tg, hLIPGTg; KO, LIPG ${ }^{-1-}$.
\end{abstract}

play after exposure to oxidized LDL (17). Our group identified LIPG through a comprehensive search for genes differentially regulated in endothelial cells during angiogenesis and identified the gene as being upregulated by suppression subtraction hybridization during tube formation on Matrigel (16). The EL predicted protein sequence encoded all of the expected lipase motifs, including the catalytic triad of serine, aspartate, and histidine, as well as heparin and lipid binding regions. The lid region, the domain felt to contribute to substrate specificity, was found to be different from those of HL and LPL, suggesting a different functional profile. Indeed, EL was shown to have relatively more phospholipase activity than triglyceride lipase activity in in vitro assays. The expression pattern of EL was also distinct from the other family members, being found in thyroid, lung, placenta, kidney, liver, and testis as well as adult vasculature. EL is expressed in vivo by endothelial cells, smooth-muscle cells, and macrophages in the arterial vessel wall, and cell culture experiments have suggested that LIPG is highly regulated by factors such as biophysical forces and cytokines that are known to be involved in vascular disease processes (18, and our unpublished data).

The existence of EL had not been predicted by studies of lipid metabolism. Careful evaluation of the function of this new lipase is thus critically important to establish its role within the context of the known lipase family of molecules. Preliminary functional information was provided by in vivo adenoviral gene transfer experiments that indicated EL overexpression lowered HDL levels (17). Accordingly, we have established a number of mouse models on the $\mathrm{C} 57 \mathrm{Bl} / 6$ background to investigate the actions of this new lipase family member in a rigorous fashion. Gene targeting in ES cells was employed to functionally delete the gene, and this allele was introduced into the germline and shown to be a functional null by RNA expression studies. The allele was bred from the $129 / \mathrm{C} 57 \mathrm{Bl} / 6$ mixed background to a homogeneous $\mathrm{C} 57 \mathrm{Bl} / 6$ background. In addition, a BAC clone carrying the entire human LIPG locus was used to generate $\mathrm{C} 57 \mathrm{Bl} / 6$ mice transgenic for this locus, and these mice were characterized with respect to transgene expression.

A distinct role for EL regulation of HDL cholesterol is documented, with both knockout and transgenic mouse models showing a correlation between functional EL expression levels and HDL cholesterol. Decreased expression of native EL in mice showed a gene dosage effect, with HDL cholesterol increasing in relation to the number of null alleles. In male $L I P G^{-/-}$ mice, there was a $57 \%$ increase in HDL cholesterol as compared with wild-type controls. HDL cholesterol levels in transgenic animals that expressed modest levels of human EL were correspondingly decreased, suggesting that pronounced overexpression of EL is not required to alter HDL levels. Measurements on isolated lipoprotein fractions also documented the correlation between EL expression and cholesterol level in HDL particles. There were observed differences between the biochemical and the FPLC lipid analyses, with the HDL peak fractions from FPLC showing less of an increase in HDL cholesterol in knockout animals but greater decreases in HDL cholesterol in the transgenic animals. This discrepancy between the levels determined by biochemical methods, which remove apoB-containing lipoproteins to measure HDL cholesterol, and FPLC, which separates the lipoproteins by size, could be due to the presence of small LDL particles in the HDL range. Indeed, SDS-PAGE analysis of FPLC lipoprotein fractions identified apoB in the HDL fractions, indicating the presence of some LDL particles, as hypothesized.

HDL particle composition analysis on plasma fractionated by both FPLC and ultracentrifugation provided support for the hypothesis that the increase in HDL cholesterol in knockout animals was a function of an increased number of particles rather than modification of the composition of HDL particles. Quantitative SDS-PAGE and Western blotting of FPLC fractions revealed that apoA-I was the dominant protein in the HDL particles and that there was a concomitant increase in apoA-I in HDL fractions. These data also confirmed that the increased cholesterol peak on FPLC for the LIPG knockout animals was associated with HDL particles and that there was no shift in particle size. The increase in apoA-I in the knockout animals was confirmed by SDS-PAGE analysis of HDL density fractions from the ultracentrifugation. Corresponding data with transgenic animals revealed decreased apoA-I levels that tracked with decreased HDL cholesterol. 
Quantitation of the protein, cholesterol, and phospholipid components of the density fraction corresponding to HDL revealed that the relative contribution of each of these components to the HDL particle was unchanged in the knockout and transgenic animals as compared with the controls. Chromatography of the HDL density fraction revealed that the phospholipid makeup of the HDL particles was unchanged and that there was not an enrichment with Lyso-PC. All of these data are consistent with an overall change in HDL particle number resulting from changes in EL levels in these genetic models.

The in vivo enzymatic activity described here is in keeping with the in vitro functional data that have been obtained with recombinant EL protein. Such data have indicated that EL has minimal triglyceridase activity but functions primarily as a phospholipase $(16,17,19)$. Significant increases in triglyceride levels in female knockout mice and trends toward decreased triglyceride levels in male transgenic animals suggests that EL may have some in vivo triglyceridase activity, as suggested by McCoy et al. (19). However, the changes in HDL levels noted in the genetic mouse lines was much greater. This is consistent with the observation that HDL is the lipoprotein class that is affected most by the level of EL expression. Phospholipase activity in postheparin plasma correlated with the level of EL expression. It was significantly increased in transgenic animals and decreased in knockout animals. There was a large amount of phospholipase activity contributed by circulating lipases in the mice - presumably primarily HL, which is not bound to endothelial cell surfaces in mice - and this did not change with manipulation of EL expression. However, the loss of EL in the homozygous knockout animals resulted in a significant decrease in the postheparin augmentation of phospholipase activity. These data clearly point to EL as contributing the majority of heparin-releasable phospholipase activity in mice and thus having a prominent role contributing to phospholipase activity in vivo.

Overall, findings from these experiments employing genetic models are in agreement with, and significantly extend, previous functional studies. Together, these data support the hypothesis that EL makes a physiological contribution to HDL metabolism in mice. Given the known difference in HDL lipoprotein species between mice and humans, observations made here with genetic mouse models will require confirmation in humanized mice and humans. If EL has a similar role in humans, its regulation by pathophysiological effectors will establish EL as an important independent regulator of HDL levels. At this time, the mechanism by which EL modulates HDL level is unclear. If EL hydrolyzes HDL phospholipids, it would necessitate removal of the other components of the HDL particle such as apoA-I and cholesterol by transfer to tissue or other lipoprotein classes. This should result in an increased rate of catabolism of all of the components of HDL and would be consistent with the observation that apoA-I and HDL cholesterol levels fall proportionately with HDL phospholipid.

\section{Acknowledgments}

This work was supported by the Donald W. Reynolds Cardiovascular Clinical Research Center at Stanford University and by grant DK38318 to A.D. Cooper, by grant DK38107 from the Stanford University Digestive Diseases Center to A.D. Cooper, and by a grant from the American Heart Association to S. Choi.

1. Castelli, W.P., et al. 1977. HDL cholesterol and other lipids in coronary heart disease. The cooperative lipoprotein phenotyping study. Circulation. 55:767-772.

2. Gordon, T., Castelli, W.P., Hjortland, M.C., Kannel, W.B., and Dawber, T.R. 1977. High density lipoprotein as a protective factor against coronary heart disease. The Framingham Study. Am. J. Med. 62:707-714.

3. Heller, D.A., de Faire, U., Pedersen, N.L., Dahlen, G., and McClearn, G.E. 1993. Genetic and environmental influences on serum lipid levels in twins. N. Engl. J. Med. 328:1150-1156.

4. Hasstedt, S.J., et al. 1984. The inheritance of high density lipoprotein cholesterol and apolipoproteins A-I and A-II. Atherosclerosis. 51:21-29.

5. Rader, D.J., and Ikewaki, K. 1996. Unravelling high density lipoproteinapolipoprotein metabolism in human mutants and animal models. Curr. Opin. Lipidol. 7:117-123.

6. Qin, S., et al. 2000. Phospholipid transfer protein gene knock-out mice have low high density lipoprotein levels, due to hypercatabolism, and accumulate apoA-IV-rich lamellar lipoproteins. J. Lipid Res. 41:269-276.

7. Libby, P. 2001. Managing the risk of atherosclerosis: the role of high-density lipoprotein. Am.J. Cardiol. 88:3N-8N.

8. Oram, J.F., and Lawn, R.M. 2001. ABCA1. The gatekeeper for eliminating excess tissue cholesterol. J. Lipid Res. 42:1173-1179.

9. Choi, S.Y., Komaromy, M.C., Chen, J., Fong, L.G., and Cooper, A.D. 1994. Acceleration of uptake of LDL but not chylomicrons or chylomicron remnants by cells that secrete apoE and hepatic lipase. J. Lipid Res. 35:848-859.

10. Goldberg, I.J. 1996. Lipoprotein lipase and lipolysis: central roles in lipoprotein metabolism and atherogenesis. J. Lipid Res. 37:693-707.

11. Goldberg, I.J., and Merkel, M. 2001. Lipoprotein lipase: physiology, biochemistry, and molecular biology. Front. Biosci. 6:D388-D405.

12. Shimada, M., et al. 1996. Suppression of diet-induced atherosclerosis in low density lipoprotein receptor knockout mice overexpressing lipoprotein lipase. Proc. Natl. Acad. Sci. USA. 93:7242-7246.

13. Yagyu, H., et al. 1999. Overexpressed lipoprotein lipase protects against atherosclerosis in apolipoprotein E knockout mice. J. Lipid Res. 40:1677-1685.

14. Babaev, V.R., et al. 1999. Macrophage lipoprotein lipase promotes foam cell formation and atherosclerosis in vivo. J. Clin. Invest. 103:1697-1705.

15. Mezdour, H., Jones, R., Dengremont, C., Castro, G., and Maeda, N. 1997. Hepatic lipase deficiency increases plasma cholesterol but reduces susceptibility to atherosclerosis in apolipoprotein E-deficient mice. J. Biol. Chem. 272:13570-13575.

16. Hirata, K., et al. 1999. Cloning of a unique lipase from endothelial cells extends the lipase gene family. J. Biol. Chem. 274:14170-14175.

17. Jaye, M., et al. 1999. A novel endothelial-derived lipase that modulates HDL metabolism. Nat. Genet. 21:424-428.

18. Hirata, K., Ishida, T., Matsushita, H., Tsao, P.S., and Quertermous, T. 2000. Regulated expression of endothelial cell-derived lipase. Biochem. Biophys. Res. Commun. 272:90-93.

19. McCoy, M.G., et al. 2002. Characterization of the lipolytic activity of endothelial lipase. J. Lipid Res. 43:921-929.

20. Pennacchio, L.A., et al. 2001. An apolipoprotein influencing triglycerides in humans and mice revealed by comparative sequencing. Science. 294:169-173.

21. Ishida, T., et al. 1999. Identification of mRNA for 5-HT1 and 5-HT2 receptor subtypes in human coronary arteries. Cardiovasc. Res. 41:267-274.

22. Jiao, S., Cole, T.G., Kitchens, R.T., Pfleger, B., and Schonfeld, G. 1990. Genetic heterogeneity of lipoproteins in inbred strains of mice: analysis by gel-permeation chromatography. Metabolism. 39:155-160.

23. Havel, R.J., Eder, H.A., and Bragdon, H.J. 1955. Distribution and chemical composition of ultracentrifugally separated lipoproteins in human serum. J. Clin. Invest. 34:1345-1353.

24. Bligh, E.G., and Dyer, W.J. 1959. A rapid method of total lipid extraction and purification. Can. J. Biochem. Physiol. 37:911-1017.

25. Nakagawa, Y., Setaka, M., and Nojima, S. 1991. Detergent-resistant phospholipase A1 from Escherichia coli membranes. In Methods in Enzymology. E.A. Dennis, editor. Academic Press. San Diego, California, USA. 309-315. 